Original Article

\title{
Patellar Tendon Reconstruction Utilizing Autograft Hamstring: A Docking Technique for the Sports Medicine Surgeon
}

\author{
Brian M Leo, $M D^{1^{*}}$ and Michael $K$ Krill, $M D, A T C^{2,3}$ \\ ${ }^{1}$ Department of Orthopaedic Surgery, Cleveland Clinic Florida, Weston, FL, USA \\ ${ }^{2}$ Charles E Schmidt College of Medicine, Florida Atlantic University, Boca Raton, FL, USA \\ ${ }^{3}$ Department of Neurology, Washington University in Saint Louis, Physical Medicine \& Rehabilitation, St Louis, MO, USA
}

\begin{abstract}
Patellar tendon ruptures are a relatively uncommon injury and account for roughly $3 \%$ of all injuries to the tendon ligament complex of the knee. Numerous techniques, grafts, augmentations and sutures utilized intraoperatively to repair the patellar tendon have been previously studied. Management of a failed primary repair or chronic patellar tendon tear can be challenging with high complication rates due to infection, wire breakage and tibial fracture with a high number of patients unable to return to pre-injury activity levels. These less than ideal long-term outcomes create uncertainty for surgeons when choosing the appropriate repair technique. We present a case report to describe a surgical technique using autograft tissue with common instrumentation, implants and techniques familiar to sports surgeons. This docking strategy for challenging patients alleviates concern for the required length of the autograft hamstring tendon and repair strength following a recent patellar tendon reconstruction failure. The surgeon should feel confident of secure fixation and the ability to achieve early rehabilitation with the potential for long-term success.
\end{abstract}

\section{Keywords}

Patellar tendon, Rupture, Reconstruction, Autograft, Hamstring tendon

\section{Introduction}

Patellar tendon ruptures are a relatively uncommon injury and account for roughly $3 \%$ of all injuries to the extensor ligament complex of the knee [1-8]. Several studies recently reported a good prognosis following patellar tendon rupture if the diagnosis and primary surgical repair is completed acutely, described as less than two weeks $[1,9]$. This reiterates the importance of early evaluation, diagnosis and appropriate surgical treatment to restore the extensor mechanism [1,3,912]. Studies regarding the best way to manage patellar tendon reconstruction when primary repair is not possible are vague with outcomes ranging across the entire spectrum from good to poor $[2,5,7,9,13]$.

Numerous techniques, grafts, augmentation and sutures utilized to repair the patellar tendon have been previously described in the literature [1,2,5-7,9,10,14-23]. For acute repairs, a primary repair alone or a repair utilizing Dall-Miles or non-absorbable sutures demonstrates the lowest failure and complication rates $[5,6]$. For acute repairs utilizing primary or autogenous graft techniques, the revision rates or failures, all documented as re-ruptures, are between 2-7\% [9].

The management of a failed primary repair or chronic patellar tendon tear can be devastating for a patient and a surgical challenge to achieve acceptable clinical outcomes
$[2,9]$. Revision patellar tendon surgery or surgery of a chronic tear unable to be primarily repaired has led to a wider array of repair techniques $[5,7,9]$. Unfortunately, studies evaluating reconstruction of a chronic patellar tendon rupture are frequently on a small scale with only a handful of subjects resulting in numerous treatment option recommendations $[5,9]$. A reconstruction augmented with cerclage exhibits high rates of complication including infection, wire breakage, or tibial fracture [5]; however, it does not require weeks of postsurgical immobilization, and after two years, displays similar range of motion when compared bilaterally $[10,24]$. The most often utilized graft to reconstruct the patellar tendon is autologous tissue; either semitendinosus, gracilis or both $[2,5,9]$. A retrospective review by Maffulli, et al., evaluated 18

*Corresponding author: Brian M Leo, MD, Department of Orthopaedic Surgery, Orthopaedic and Rheumatologic Center, Sports Health, Cleveland Clinic Florida, 2950 Cleveland Clinic Blvd, Weston, FL 33331, USA, Tel: 954-659-5430, Fax: 954-659-5423

Accepted: April 03, 2019

Published online: April 05, 2019

Citation: Leo BM, Krill MK (2019) Patellar Tendon Reconstruction Utilizing Autograft Hamstring: A Docking Technique for the Sports Medicine Surgeon. J Orthop Surg Tech 2(1):68-73 
patients (11 self-described manual laborers) who underwent chronic patellar tendon reconstruction utilizing autogenous ipsilateral semitendinosus and gracilis tendons who were able to return to pre-injury work activities supporting the effectiveness of a soft tissue reconstruction [5]. Another main goal of treatment is the ability of the patient to return to pre-injury athletic activity. From patient reported surveys, Maffulli, et al. found $5 / 8(62.5 \%)$ return to the same preinjury level of recreational sport activities, two (25\%) felt uncomfortable with their knee, and one (12.5\%) abandoned recreational sport activities due to persistent knee pain [5]. These outcomes are less than ideal. As a result, patellar tendon reconstruction is quite complicated, and the level of tissue disruption and patient presentation varies between cases $[14,15,25]$. The variability of tissue disruption and numerous techniques demonstrates that the best approach may not be appropriate for every case $[14,15,25]$.

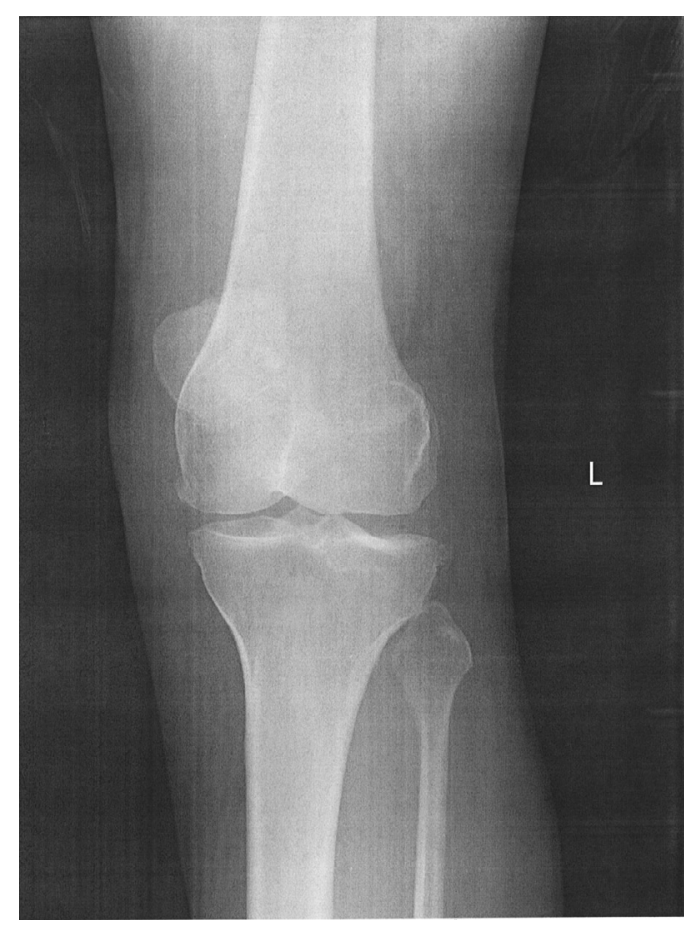

Figure 1: Pre-operative anteroposterior radiograph.
Here we describe our technique utilizing modern techniques familiar to knee surgeons to reconstruct a failed primary repair (Figure 1 and Figure 2) following additional trauma or a chronic tear requiring augmentation. We present this technique as an additional option for surgeons when handling a challenging case with limited remnant tissue following a recent re-rupture of a primary patellar tendon reconstruction.

\section{Surgical Technique}

The patient is positioned supine and placed under general anesthesia. A midline incision from the superior patellar pole to the medial aspect of the tibial tubercle is made. After exposure of the tendon confirming the need to augment and reconstruct the patellar tendon, the sartorial fascia is elevated as done during autograft hamstring anterior cruciate ligament reconstruction and the semitendinosus and gracilis

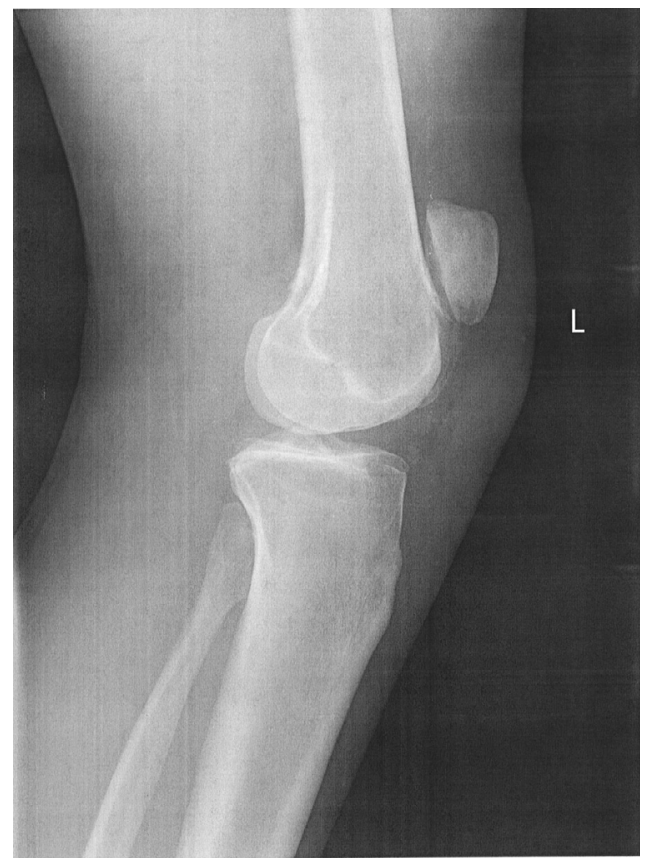

Figure 2: Pre-operative lateral radiograph demonstrating patella alta.

Table 1: Surgical Technique.

1. Autograft gracilis and semitendinosus hamstring tendons are harvested in the standard fashion using a closed-ended tendon stripper, cleaned of muscle tissue and whip-stitched with a \# non-absorbable suture at each end

2. A $6.5 \mathrm{~mm}$ transverse tibial tunnel is created using a guidewire $1 \mathrm{~cm}$ posterior to the tibial tubercle

3. Any remaining healthy patellar tendon is preserved

4. The semitendinosus and gracilis tendons are passed through the transverse tibial tunnel

5. Semitendinosus autograft is delivered to the inferior pole of the patella in a crisscrossed pattern and weaved into the remnant of healthy patellar tendon

6. Gracilis autograft is delivered to the inferior pole of the patella in a U-shaped pattern

7. Autograft tissue is docked into two $6.5 \mathrm{~mm}$ by $20 \mathrm{~mm}$ patellar sockets in the inferior lateral aspects of the patella and secured with BioCompression screws

8. Inferior medial patellar socket is secured first before moving to the lateral patellar socket

9. A $6.25 \mathrm{~mm}$ Bio-Compression screw provides interference fixation in the transverse tibial tunnel 
tendons are harvested in standard fashion utilizing a tendon stripping device. The autologous hamstring tissue is stripped of muscle and whip stitched at each end with a \#2 nonabsorbable suture (Table 1 ).

Using a guidewire $1 \mathrm{~cm}$ posterior to the tibial tubercle, a $6.5 \mathrm{~mm}$ transverse tibial tunnel is created. The inferior pole of the patella is prepared, and healthy patellar tendon is preserved to overlie the autograft reconstructive tissue. The semitendinosus and gracilis tendons are passed through the transverse tibial tunnel with the aid of a Hewson suture passer or shuttling stitch (Figure 3 ). The semitendinosus autograft is delivered proximally in a criss crossed pattern and weaved into the remnant of the patellar tendon (Figure 4). The gracilis autograft is positioned in a U-shaped pattern passed through the transverse tibial tunnel and guided towards the remnant of the patellar tendon. Two $6.5 \mathrm{~mm}$ by $20 \mathrm{~mm}$ patellar sockets are created in the patella on the inferior lateral aspects for the tenodesis implant.

With the knee held at 20 degrees of flexion one end of the gracilis autograft and one end of the semitendinosus

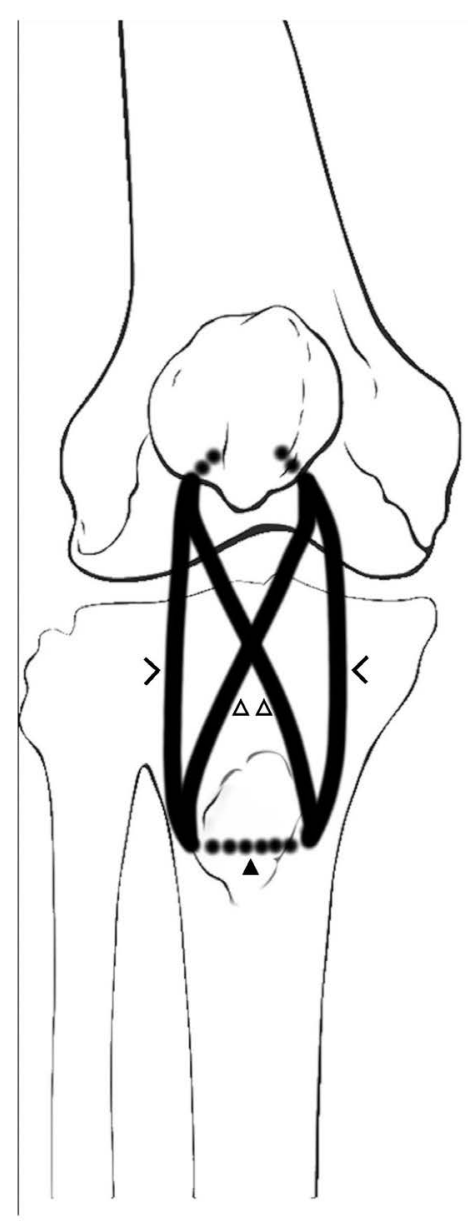

Figure 3: The autograft semitendinosus and gracilis tendons are passed through a $6.5 \mathrm{~mm}$ transverse tibial tunnel (solid black arrows). The semitendinosus is positioned in a criss crossed pattern (hollow white arrows) weaved in with the remnant of the patellar tendon. The gracilis is positioned in a U-shaped pattern (guillemets). Both tendons are passed to the inferior patellar sockets for the tenodesis implant. autograft is passed into the inferior medial patellar socket and secured in place with a $6.25 \mathrm{~mm}$ Bio-Compression screw (Arthrex, Naples, FL). The free end of the graft tails is placed under tension and advanced into the lateral patellar socket and fixated with a second $6.25 \mathrm{~mm}$ Bio-Compression screw. Additional interference fixation is placed in the transverse tibial tunnel utilizing a $6.25 \mathrm{~mm}$ Bio-Compression screw (Arthrex, Naples, FL) to fixate the graft and prevent graft abrasion in the osseous tunnel. The knee is then taken through a full range of motion to confirm no disruption of the reconstructed tendon. The proximal and distal remnants of the patellar tendon are sewn into the autograft hamstring tissue using nonabsorbable \#2 polyethylene/polyester sutures (Arthrex, Naples, FL). Additionally, with the knee at 30 degrees of flexion, a nonabsorbable \#5 polyethylene/polyester suture (Arthrex, Naples, FL) is used to cerclage through the knee retinaculum and quadriceps tendon proximal to the patella and distal through the patella tendon reconstruction.

Patients are discharged the day of surgery with cryotherapy and a knee immobilizer with $50 \%$ partial weight bearing (PWB). Passive range of motion (ROM) from $0^{\circ}-30^{\circ}$ is initiated and maintained for the first two weeks. From two to six weeks post-operatively, patients continue with $50 \%$ PWB in full extension and slowly advance passive ROM up to $90^{\circ}$. Patients then begin to weight bear as tolerated 6 weeks after surgery, wean off crutches and progress ROM passively and begin active assistive motion. Light resistance is begun 12 weeks post-operatively and release to unrestricted activity 6 months after surgery.

\section{Case Patient}

The described procedure has been performed on two patients. The second patient was lost to follow up at six months but had no complaints or complications during his known post-operative course. The first patient to undergo the procedure was a 45-year-old male who had undergone an initial patellar tendon reconstruction in 2013. Just under two months after primary reconstruction, the patient had

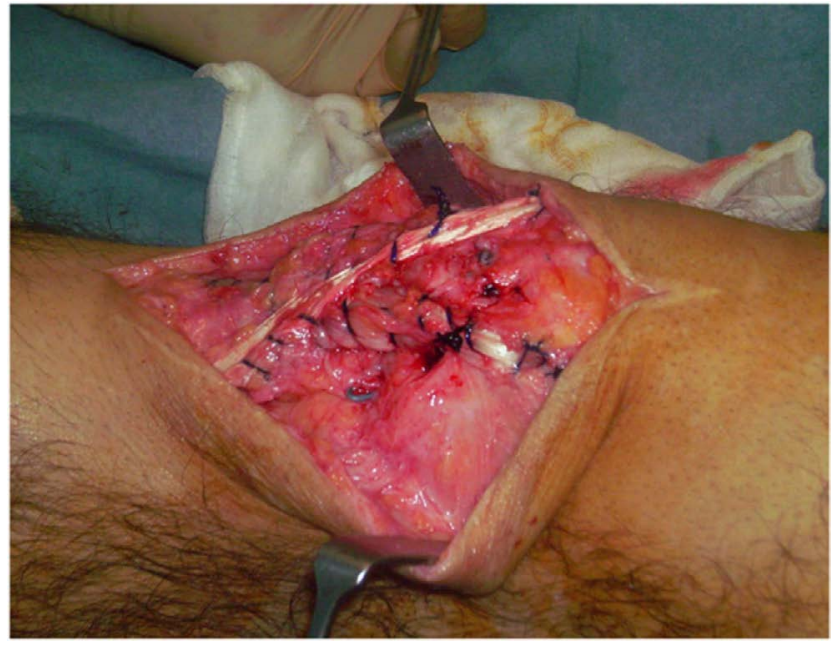

Figure 4: Intra-operative visualization of the final criss crossed appearance of the autograft semitendinosus tendon weaved in with the remnant patellar tendon. 


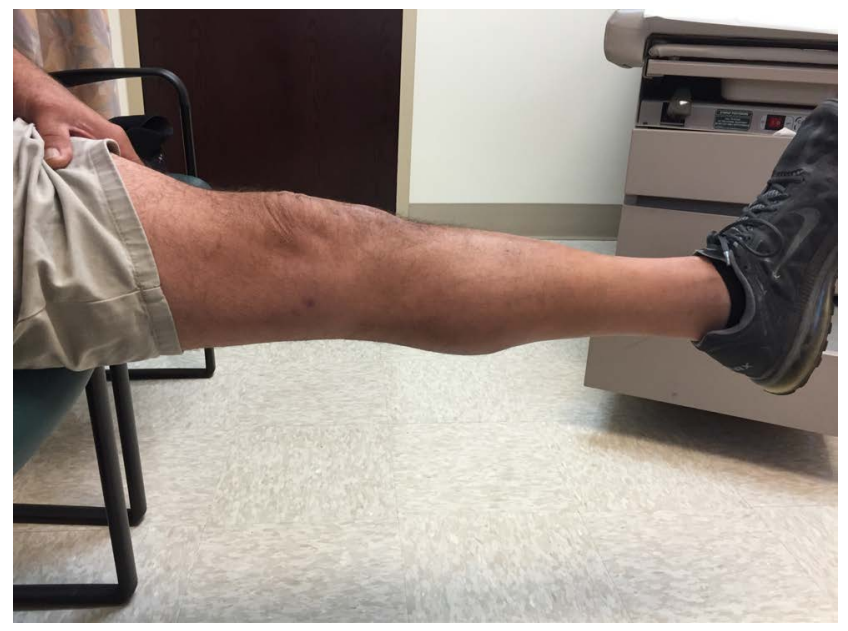

Figure 5: Post-operative follow-up picture at one-year with the patient demonstrating full extension.

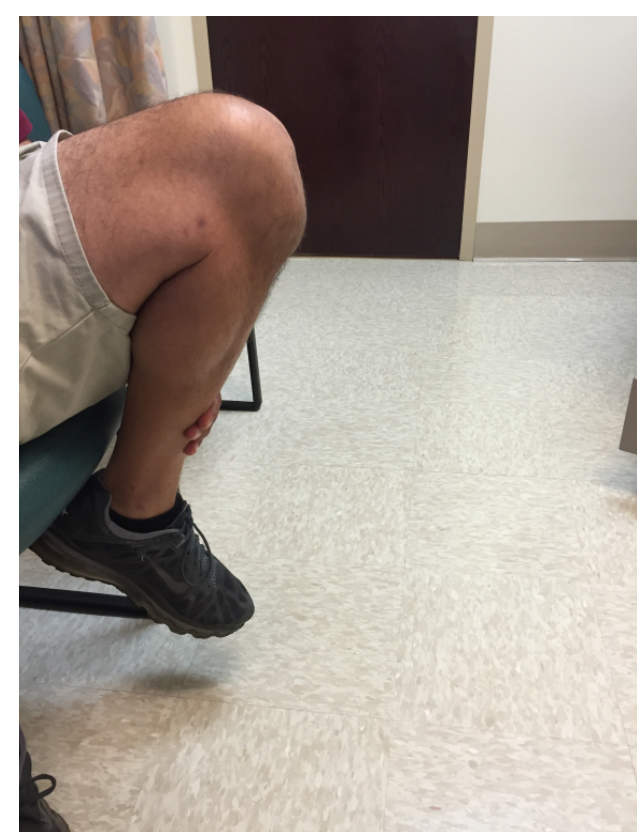

Figure 6: Post-operative follow-up picture at one-year with the patient demonstrating flexion.

a mechanical fall down the stairs at home that resulted in a re-rupture of the repair. The native repaired tendon was macerated, and the intraoperative decision was that two grafts were necessary for an adequate reconstruction using the technique described above. The patient had follow-up at 2 weeks, 6 weeks, 12 weeks, 6 months, 1 year. In addition, he followed up as needed afterward and reported no complaints at two years post-revision. The patient was able to return to his pre-injury level of physical activity and his major limitation was pain secondary to osteoarthritis in his contralateral knee. He reports no residual knee pain with his surgically-repaired knee. Overall, he was extremely pleased with the results of the reconstruction (Figure 5 and Figure 6).

\section{Discussion}

Early primary surgical repair of an acute patellar tendon

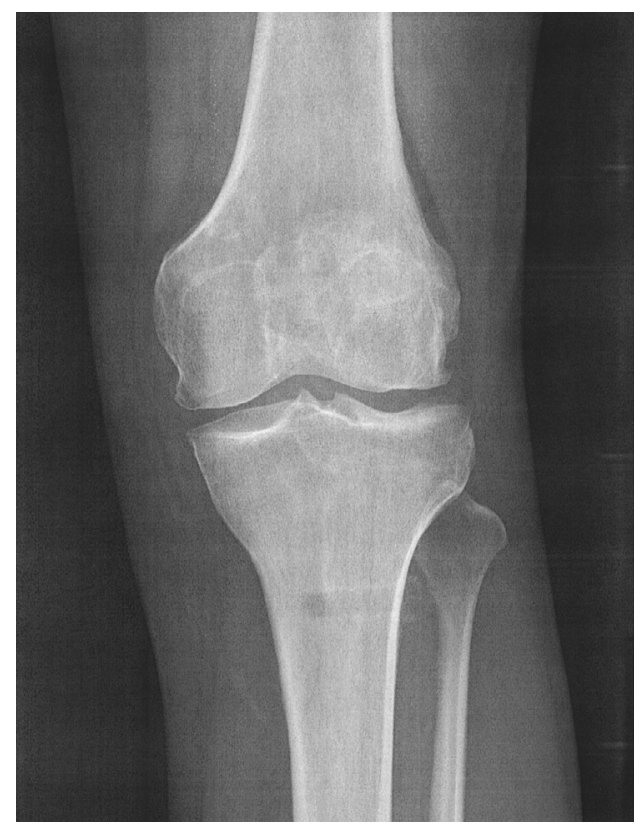

Figure 7: Post-operative anteroposterior radiograph demonstrating patella alignment after reconstruction.

rupture has been shown to have the best post-operative outcomes [11]; however, a surgical challenge is presented for delayed repairs, mid-substance tears with poor remaining tissue quality or after failed primary repairs. These challenges require tendon reconstruction or augmentation to re-establish the extensor mechanism and improve patient quality of life with a hopeful return to their pre-injury activity level. Multiple techniques, grafts, sutures and augmentations have been described previously for reconstruction [1,2,5$7,9,10,14,15]$. To our knowledge, the integrity and histological characteristics of the hamstring autograft has not been described for extra-articular knee procedures. In regard to anterior cruciate ligament $(A C L)$ reconstructions, age and gender play a role in the graft-selection decision-making process $[26,27]$. There are well-documented increased rates of allografts compared to autograft tissue on widely research $A C L$ reconstructions $[26,28]$. Gangliano, et al. determined that the structure of aging tendons is preserved, and aging tenocytes maintained their ability of extracellular matrix remodeling when evaluating semitendinosus and gracilis tendons in older patient populations (mean group ages of 41.8 versus 72.7 years old) [29]. This may result in trying to maintain the remnant tissue during reconstruction. The role of gender on the healing and maturation process of hamstring autografts is still unclear. The difficulty of extrapolating the findings from other studies exists as many are $A C L$ or other intraarticular reconstructions and not extraarticular in nature. We describe our technique utilizing autograft hamstring, common techniques and familiar implants commonly used and reproducible by sports medicine surgeons that has provided our patients with a successful reconstruction of the patellar tendon and extensor mechanism (Figures 7 and Figure 8).

Importantly and differentiating our technique from other published reports is the length of autograft hamstring tendon 


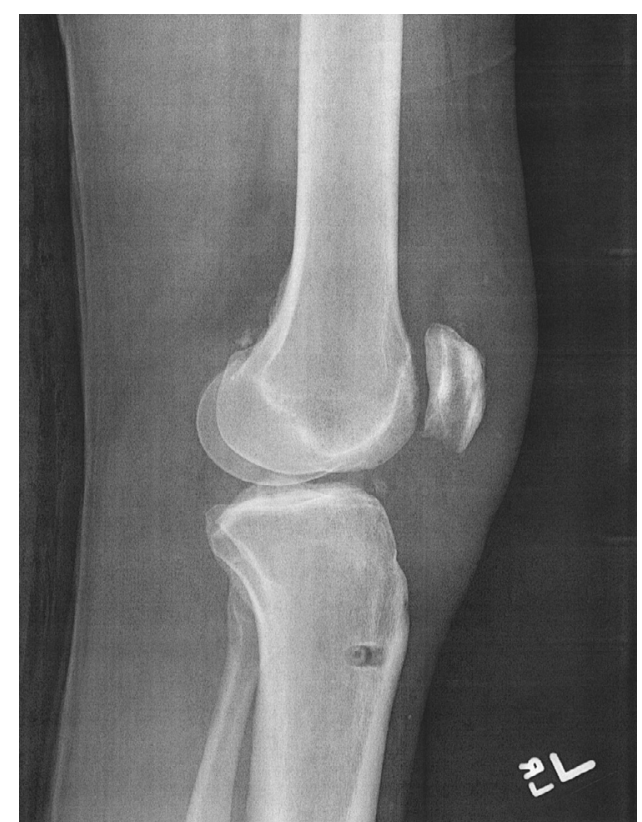

Figure 8: Post-operative lateral radiograph demonstrating patella alignment after reconstruction.

and is less of a concern with this tendon docking procedure because the graft is not required to be pulled the full length through a transpatellar tunnel for fixation as others have described $[1,2,4,7,9]$. The surgeon should feel confident with this reconstructive patellar tendon reconstruction to allow for secure fixation and early rehabilitation to achieve knee range of motion and independent ambulation.

\section{Limitations}

Due to the low incidence rate of patellar tendon reconstruction and studies with small sample sizes, it is challenging to make large scale recommendations on the outcomes of the technique itself. In addition, there were no evidence-based outcome measurements. Further follow-up and continued research into the presented technique may help provide a clearer protocol for chronic patellar tendon reconstruction.

\section{Summary}

Primary patellar tendon repair for acute traumatic patellar tendon ruptures is the desired repair. However, we have had clinical success with a transtibial autograft hamstring patellar docking technique for patellar tendon reconstruction for challenging patients utilizing techniques and implants familiar to sports medicine knee surgeons.

\section{Funding}

None.

\section{Conflicts of Interest}

The authors declare no conflicts of interest. The authors do not have any proprietary interests in the materials described in the article. The first author, Dr. Brian M. Leo, is a consultant for Cayenne Medical - Zimmer Biomet.

\section{References}

1. Roudet A, Boudissa M, Chaussard C, et al. (2015) Acute traumatic patellar tendon rupture: Early and late results of surgical treatment of 38 cases. Orthop Traumatol Surg Res 101: 307-311.

2. Abdou YE (2014) Reconstruction of a chronic patellar tendon rupture with semitendinosus autograft. Arch Orthop Trauma Surg 134: 1717-1721.

3. Flanigan DC, Bloomfield M, Koh J (2011) A biomechanical comparison of patellar tendon repair materials in a bovine model. Orthopedics.

4. Grim C, Lorbach O, Engelhardt M (2010) Quadriceps and patellar tendon ruptures. Orthopade 39: 1127-1134.

5. Maffulli N, Del Buono A, Loppini M, et al. (2013) Ipsilateral hamstring tendon graft reconstruction for chronic patellar tendon ruptures: Average 5.8-year follow-up. J Bone Jt Surg-Am 95: e1231-e1236.

6. Marder RA, Timmerman LA (1999) Primary repair of patellar tendon rupture without augmentation. Am J Sports Med 27: 304-307.

7. Spoliti M, Giai Via A, Padulo J, et al. (2016) Surgical repair of chronic patellar tendon rupture in total knee replacement with ipsilateral hamstring tendons. Knee Surg Sports Traumatol Arthrosc 24: 3183-3190.

8. Ibounig T, Simons TA (2015) Etiology, diagnosis and treatment of tendinous knee extensor mechanism injuries. Scand J Surg 105: 67-72.

9. Gilmore JH, Clayton-Smith ZJ, Aguilar M, et al. (2015) Reconstruction techniques and clinical results of patellar tendon ruptures: Evidence today. The Knee 22: 148-155.

10. Schliemann B, Grüneweller N, Yao D, et al. (2016) Biomechanical evaluation of different surgical techniques for treating patellar tendon ruptures. Int Orthop 40: 1717-1723.

11. Zhang J, Keenan C, Wang JH (2013) The effects of dexamethasone on human patellar tendon stem cells: Implications for dexamethasone treatment of tendon injury. J Orthop Res 31: 105-110.

12. Phillips K, Costantino TG (2014) Diagnosis of patellar tendon rupture by emergency ultrasound. J Emerg Med 47: 204-206.

13. Tejwani NC, Lekic N, Bechtel C, et al. (2012) Outcomes after knee joint extensor mechanism disruptions: Is it better to fracture the patella or rupture the tendon? J Orthop Trauma 26: 648-651.

14. Wiegand N, Naumov I, Vamhidy L, et al. (2013) Reconstruction of the patellar tendon using a Y-shaped flap folded back from the vastus lateralis fascia. The Knee 20: 139-143.

15. Bek D, Demiralp B, Kömürcü $M$, et al. (2008) Neglected patellar tendon rupture: a case of reconstruction without quadriceps lengthening. J Orthop Traumatol 9: 39-42.

16. Chloros GD, Razavi A, Cheatham SA (2014) Complete avulsion of the patellar tendon from the tibial tubercle in an adult without predisposing factors. J Orthop Sci 19: 351-353.

17. El-Desouky II, Mohamed MM, Al Assassi M (2014) Primary repair of ruptured patellar tendon augmented by semitendinosus. J Knee Surg 27: 207-213.

18. Jablonski JJ, Jarmuziewicz P, Druzbicki M (2011) Reconstruction of chronic patellar tendon rupture with semitendinosus tendon: case report. Ortop Traumatol Rehabil 13: 607-615.

19. Karas V, Sherman S, Hussey K, et al. (2014) Allograft reconstruction 
for extensor mechanism injuries. J Knee Surg 27: 489-496.

20. Naim S, Gougoulias N, Griffiths D (2011) Patellar tendon reconstruction using LARS ligament: Surgical technique and case report. Strateg Trauma Limb Reconstr 6: 39-41.

21. Sundararajan SR, Srikanth KP, Rajasekaran S (2013) Neglected patellar tendon ruptures: A simple modified reconstruction using hamstrings tendon graft. Int Orthop 37: 2159-2164.

22. Takazawa $Y$, Ikeda H, Ishijima M, et al. (2013) Reconstruction of a ruptured patellar tendon using ipsilateral semitendinosus and gracilis tendons with preserved distal insertions: two case reports. BMC Res Notes.

23. Vadala A, lorio R, Bonifazi AM, et al. (2012) Re-revision of a patellar tendon rupture in a young professional martial arts athlete. J Orthop Traumatol 13: 167-170.

24. Ramseier LE, Werner CML, Heinzelmann M (2006) Quadriceps and patellar tendon rupture. Injury 37: 516-519.
25. Volk WR, Yagnik GP, Uribe JW (2014) Complications in brief: Quadriceps and patellar tendon tears. Clin Orthop 472: 10501057.

26. Group M (2015) Factors Influencing Graft Choice in Revision Anterior Cruciate Ligament Reconstruction in the MARS Group. J Knee Surg 29: 458-463.

27. Thaunat M, Fayard JM, Sonnery-Cottet B (2019) Hamstring tendons or bone-patellar tendon-bone graft for anterior cruciate ligament reconstruction? Orthop Traumatol Surg Res 105: S89-S94.

28. Nissen KA, Eysturoy NH, Nielsen TG, et al. (2018) Allograft use results in higher re-revision rate for revision anterior cruciate ligament reconstruction. Orthop J Sports Med 6: 232596711877538.

29. Gagliano N, Menon A, Martinelli C, et al. (2013) Tendon structure and extracellular matrix components are affected by spasticity in cerebral palsy patients. Muscles Ligaments Tendons J 3: 42-50. 